\title{
The Research of Fault Diagnosis with Time-Delay Switched Systems
}

\author{
H.Y. Sun \\ Hebei Vocational \& Technical College of Building Materials
}

China

\begin{abstract}
The time delay switched system fault diagnosis control is proposed based on LMI skill. The observer is designed based on Lyapunov stability theorem with switched system, and the LMI form of uniformly bounded stability result for fault estimation error system is given. Finally, the simulation is carried by MATLAB/SIMULINK software, to verify the feasibility of the proposed algorithm.
\end{abstract}

Keywords- switched systems; fault diagnosis; LMI

\section{INTRODUCTION}

The main reason for fault diagnosislaid which increasing demand for high reliability and safety in many actual engineering control systems [1-3]. A lot of fruitful results can be found recently, most design schemes of fault diagnosis architectures for dynamic system with linear system, nonlinear system and switched system [4-6]. For the switched systems, which exist in the practical systems widely, and the switched phenomenon exists in industrial control nature or in the life of people widely. Unavoidable modeling uncertainties, which arise due to modeling errors and time delay, then the adaptive control method, can be found in this subject [7-8]. So the fault diagnosis research of switched systems with time-delay has attracted the attention of many scholars. Motivated by these considerations, the fault diagnosis with delay switched system is considered, the observer design for fault is designed and the error system is proved by Lyapunov stability. The result in form of linear matrix inequality, and Lyapunov is applied to the strict proof. At last, the simulation of MATLAB in a numerical example proved the validity of the conclusion.

\section{SYSTEM DESCRIPTION}

Time-delay switched systems can be described as

$$
\begin{aligned}
\dot{x}(t) & =A_{\sigma(t)} x(t)+A_{d \sigma(t)} x(t-d) \\
& +B_{\sigma(t)} u(t)+E_{\sigma(t)} f(t) \\
y(t) & =C_{\sigma(t)} x(t) \\
x(\theta) & =\phi(\theta), \forall \theta \in[-d, 0]
\end{aligned}
$$

where $x(t) \in \mathfrak{R}^{n}$ express the state vectors of system, $u(t) \in \mathfrak{R}^{m}$ expresses the system input, $y(t) \in \mathfrak{R}^{p}$ is the system output, $f(t) \in \mathfrak{R}^{r}$ is the actuator fault of the system, $A_{\sigma(t)}, A_{d \sigma(t)}, B_{\sigma(t)}, C_{\sigma(t)}, E_{\sigma(t)}$ arethe system matrixes with appropriate dimension, for switching signal, $\sigma(t):[0,+\infty) \rightarrow \psi=\{1, \cdots, N\}$, where $N \geq 1$, especially, when $N=1$, system become ordinary time-delay systems, when $N>1$,it is supposedthat the i subsystem work, that is $\sigma(t)=i$,

so the formula was established

$$
A_{\sigma(t)}=A_{i}, A_{d \sigma(t)}=A_{i}, B_{\sigma(t)}=B_{i}, C_{\sigma(t)}=C_{i}, E_{\sigma(t)}=E_{i}
$$

where $\left(A_{i} \quad C_{i}\right)$ is observable, the matrix $E_{i}$ is a line full rank matrix, that is $\operatorname{rank}\left(E_{i}\right)=r$, actuator fault satisfies the norm bounded condition $\|\dot{f}(t)\| \leq f_{1},\|f(t)\| \leq f_{2}, \phi(\theta)$ is the initial function.

Lemma 1(Schur Complement lemma), for a givensymmetric matrix

$$
\boldsymbol{Q}=\left[\begin{array}{ll}
\boldsymbol{Q}_{11} & \mathbf{Q}_{12} \\
\mathbf{Q}_{12}^{T} & \mathbf{Q}_{22}
\end{array}\right]<0
$$

Where $\boldsymbol{Q}_{i i}$ is a symmetric matrix of $r_{i} \times r_{i}, r_{i}$ is a positive integer, $i=1,2$, the following conditions are

(1) $\boldsymbol{Q}<0$;

(2) $\boldsymbol{Q}_{11}<0$ and $\boldsymbol{Q}_{22}-\boldsymbol{Q}_{12}^{\mathrm{T}} \boldsymbol{Q}_{11}^{-1} \boldsymbol{Q}_{12}<0$;

(3) $\boldsymbol{Q}_{22}<0$ and $\boldsymbol{Q}_{11}-\boldsymbol{Q}_{12} \boldsymbol{Q}_{22}^{-1} \boldsymbol{Q}_{12}^{\mathrm{T}}<0$ 。

Lemma 2 For definite matrix composed of any positive constant $\boldsymbol{Z} \in \mathfrak{R}^{n \times n}, \boldsymbol{Z}=\boldsymbol{Z}^{\mathrm{T}}>0$,the scalars are $b>a \geq 0$, the vector function is $\boldsymbol{w}:[a, b] \rightarrow \mathfrak{R}^{n}$, the integral inequality was established:

$$
(b-a) \int_{a}^{b} \boldsymbol{w}^{\mathrm{T}}(s) \boldsymbol{Z} \boldsymbol{w}(s) \mathrm{d} s \geq\left(\int_{a}^{b} \boldsymbol{w}(s) \mathrm{d} s\right)^{\mathrm{T}} \boldsymbol{Z}\left(\int_{a}^{b} \boldsymbol{w}(s) \mathrm{d} s\right)
$$

\section{TIME-Delay System FAult OBSERVER DESIGN}

The observer can be design as following:

$$
\begin{aligned}
\dot{\hat{x}}(t) & =A_{\sigma(t)} \hat{x}(t)+A_{d \sigma(t)} \hat{x}(t-d)+B_{\sigma(t)} u(t)+E_{\sigma(t)} \hat{f}(t) \\
& +L\left(y-C_{\sigma(t)} \hat{x}(t)\right) \\
\hat{x}(\theta) & =\phi(\theta), \forall \theta \in[-d, 0]
\end{aligned}
$$

Definite the error state as 


$$
e_{x}=x-\hat{x}, e_{f}=f-\hat{f}
$$

Then the error system can be get as

$$
\dot{e}_{x}(t)=\left(A_{\sigma(t)}-L C\right) e_{x}(t)+A_{d \sigma(t)} e_{x}(t-d)+E_{\sigma(t)} e_{f}(t)
$$

If there are positive definite symmetric matrices $P_{i}, Q_{i}$ and an arbitrary matrix $Y_{i}$ satisfy the follow matrix inequality $\Xi<0$, then the fault estimation error system is uniformly bounded stable, where

$$
\begin{gathered}
Y_{i}=P_{i} L_{i}, E_{i}^{T} P_{i}=F_{i} C_{i} \\
\dot{\hat{f}}=-\Gamma^{-1}\left(F C e_{x}+F C \dot{e}_{x}+\sigma \hat{f}\right)
\end{gathered}
$$

The residence time of switching signal meets:

$$
T_{a}>T_{a}^{*}=\frac{\ln (\mu)}{\alpha}
$$

Then the system is asymptotically exponentially stable and there is $\mu \geq 1, P_{i} \leq \mu P_{j}, Q_{i} \leq \mu Q_{j}, Z_{i} \leq \mu Z_{j}$, where:

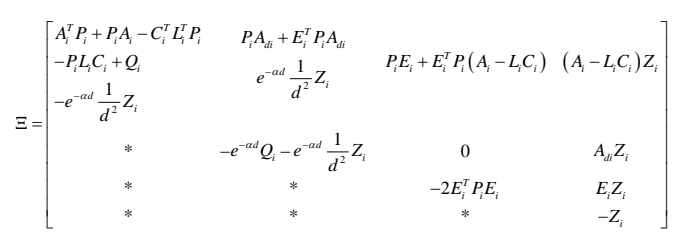

Proof: Lyapunov function is selected as following

$$
V_{i}(t)=V_{1 i}(t)+V_{2 i}(t)+V_{3 i}(t)+V_{4 i}(t)
$$

where

$$
\begin{gathered}
V_{1 i}(t)=e_{x}^{T}(t) P_{i} e_{x}(t) \\
V_{2 i}(t)=e^{-\alpha t} \int_{t-d}^{t} e^{\alpha s} e_{x}^{T}(s) Q_{i} e_{x}(s) d s \\
V_{3 i}(t)=d^{-1} e^{-\alpha t} \int_{-d t+\sigma}^{0} \int^{\alpha s} e_{x}^{T}(s) Z_{i} \dot{e}_{x}(s) d s d \sigma \\
V_{4 i}(t)=e_{f}^{T}(t) \Gamma e_{f}(t)
\end{gathered}
$$

Along (1), take the derivative of Lyapunov functional:

$$
\begin{gathered}
\dot{V}_{1 i}(t)=e_{x}^{T}(t)\left(\left(A_{i}-L_{i} C_{i}\right)^{T} P_{i}+P_{i}\left(A_{i}-L_{i} C_{i}\right)\right) e_{x}(t) \\
+2 e_{x}^{T}(t) P_{i} A_{d i} e_{x}(t-d)+2 e_{x}^{T}(t) P_{i} E_{i} e_{f}(t) \\
\dot{V}_{2 i}(t) \leq-\alpha V_{2 i}+e_{x}^{T}(t) Q_{i} e_{x}(t)-e^{-\alpha d} e_{x}^{T}(t-d) Q_{i} e_{x}(t-d) \\
\dot{V}_{3 i}(t) \leq-\alpha V_{3 i}+e_{x}^{T}(t) Z_{i} \dot{e}_{x}(t)-d^{-1} e^{-\alpha d} \int_{i=d}^{j_{i=}^{T}(s) Z_{i} \dot{e}_{x}(s) d s}
\end{gathered}
$$

Because of the system so that there is:

$$
\dot{e}_{x}^{T}(t) Z_{i} \dot{e}_{x}(t)=\xi^{T}(t)\left[\begin{array}{c}
\left(A_{i}-L_{i} C_{i}\right)^{T} \\
A_{d i}^{T} \\
E_{i}^{T}
\end{array}\right] Z_{i}\left[\begin{array}{lll}
A_{i}-L_{i} C_{i} & A_{d i} & E_{i}
\end{array}\right] \xi(t)
$$

Along (1), take the derivative of Lyapunov function $V_{4 i}(t)$ :

$$
\begin{aligned}
\dot{V}_{4 i}(t)= & -2 e_{f}^{T}(t) F_{i} C_{i} e_{x}-2 e_{f}^{T}(t) F_{i} C_{i}\left(A_{i}-L_{i} C_{i}\right) e_{x}(t) \\
& -2 e_{f}^{T}(t) F_{i} C_{i} A_{i i} e_{x}(t-d)-2 e_{f}^{T}(t) F_{i} C_{i} E_{i} e_{f}(t) \\
& -2 \sigma e_{f}^{T}(t)\left(e_{f}(t)+f^{*}\right)
\end{aligned}
$$

Because of $F C=E^{T} P$ and for the system the following inequality exists:

$$
\begin{aligned}
& -2 \sigma e_{f}^{T}(t)\left(e_{f}(t)+f^{*}\right) \leq-2 \sigma e_{f}^{T}(t) e_{f}(t) \\
& +2 \sigma e_{f}^{T}(t) f^{*} \leq-\sigma e_{f}^{T}(t) e_{f}(t)+\sigma f^{* T} f^{*}
\end{aligned}
$$

Select $\alpha=\sigma \lambda_{\min }\left(\Gamma^{-1}\right)$,soitcan be obtained:

$$
\begin{array}{r}
\dot{V}_{4 i}(t) \leq-\alpha V_{4 i}-2 e_{f}^{T}(t) E_{i}^{T} P_{i} e_{x}-2 e_{f}^{T}(t) E_{i}^{T} P_{i}\left(A_{i}-L_{i} C_{i}\right) e_{x}(t) \\
-2 e_{f}^{T}(t) E_{i}^{T} P_{i} A_{d i} e_{x}(t-d)-2 e_{f}^{T}(t) E_{i}^{T} P_{i} E_{i} e_{f}(t)+\sigma f_{2}^{2}
\end{array}
$$

As a result:

$$
\dot{V}_{i}(t) \leq-\alpha V_{i}(t)+\xi^{T} \Xi \xi+\delta
$$

If $\Xi<0$, where $\delta=\alpha f_{2}^{2}$, so the following will be got in $\left[t_{0}, t\right]$

$$
V_{i}(t) \leq-e^{-\alpha t} V_{i}\left(t_{0}\right)+\frac{\delta}{\alpha}
$$

So that, there is

$$
\begin{aligned}
V_{i}(t) & \leq-e^{-\alpha\left(t-t_{0}\right)} \mu^{\beta} V_{i}\left(t_{0}\right)+\frac{\delta}{\alpha} \\
& \leq-e^{-\left(\alpha-\frac{\ln \mu}{T_{a}}\right)\left(t-t_{0}\right)} \mu^{\beta} V_{i}\left(t_{0}\right)+\frac{\delta}{\alpha}
\end{aligned}
$$

Fault estimation error system is uniformly bounded stable.The theorem is proven.

\section{SIMULATION EXPERIMENT}

In order to verify the feasibility and effectiveness of the theoretical results, the simulation is proposed by MATLAB/SIMULINK software. To solved by LMI toolbox of Matlab, but in the theorem $E^{T} P=F C$ is required. To solve $E^{T} P-F C=0$, The following inequality is required to add into the process of simulation experiment.

$$
\text { s.t. }\left[\begin{array}{cc}
\eta I & \min \eta \\
* & E^{T} P-F C
\end{array}\right]>0
$$

Through a small enough positive number $\eta$, $E^{T} P-F C=0$ can be realized.

Subsystem 1:

$$
A_{1}=\left[\begin{array}{cc}
-1 & 0 \\
0 & -0.8
\end{array}\right], A_{d 1}=\left[\begin{array}{cc}
0.1 & 0 \\
0 & 0.2
\end{array}\right], B_{1}=\left[\begin{array}{l}
1 \\
1
\end{array}\right], E_{1}=\left[\begin{array}{l}
1 \\
1
\end{array}\right], C_{1}=\left[\begin{array}{ll}
0 & 1
\end{array}\right]
$$

Subsystem 2:

$$
A_{2}=\left[\begin{array}{cc}
-2 & 1 \\
1 & -3
\end{array}\right], A_{d 2}=\left[\begin{array}{ll}
1 & 1 \\
1 & 2
\end{array}\right], B_{2}=\left[\begin{array}{l}
1 \\
1
\end{array}\right], E_{2}=\left[\begin{array}{l}
1 \\
1
\end{array}\right], C_{2}=\left[\begin{array}{ll}
0 & 1
\end{array}\right]
$$


Through the algorithm of the above theorem, the LMI toolbox of the Matlab can carry out the simulation, we can get:

$$
L_{1}=\left[\begin{array}{c}
13.6138 \\
1.6245
\end{array}\right], L_{2}=\left[\begin{array}{c}
1.6245 \\
8.7539
\end{array}\right]
$$

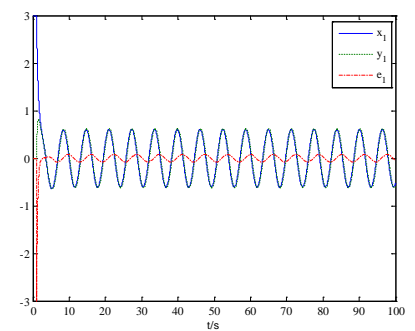

FIGURE I. STATE AND ERROR OF THE SYSTEM

As can be seen from the simulation graph I, the state of the observer $y_{1}$ can track the system state $x_{1}$ soon, the error of the $e_{1}$.

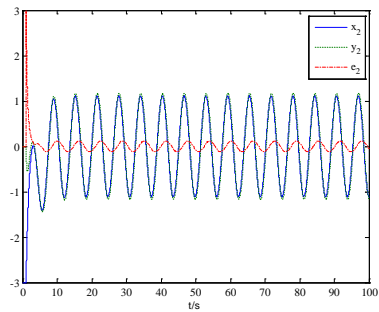

FIGURE II. STATE AND ERROR OF THE SYSTEM

As can be seen from the simulation graph II, the state of the observer $y_{2}$ can track the system state $x_{2}$ soon, the error of the $e_{2}$.

\section{CONCLUSION}

In this paper, the observer ofswitched system fault diagnosis is presented for a class of lag switched systems. The uncertain term is estimation by adaptive control method and the error system is proved to stable based on Lyapunov theory to verify the effectiveness.

\section{REFERENCES}

[1] X. Zhang. Adaptive fault-tolerant control of nonlinear uncertain systems: an information-based diagnostic approach [J]. Automatic Control, IEEE Transactions on, vol. 49, pp. 1259-1274, 2004.

[2] F. You. Active fault-tolerant control design for a class of time-delay systems [J]. pp. 5535-5538,2006.

[3] B. Marx. Robust fault-tolerant control for descriptor systems [J].Automatic Control, IEEE Transactions on, vol. 49, pp. 1869-1876, 2004.

[4] H. Yang. Observer-based fault-tolerant control for a class of switched nonlinear systems [J].Control Theory \& Applications, IET, vol. 1, pp. 1523-1532, 2007.

[5] X. Liu, S. Yuan. On Designing H [infinity] Fault Estimator for Switched Nonlinear Systems of Neutral Type [J].Communications in Nonlinear Science and Numerical Simulation, 2011.

[6] L. Zhang, H. Gao. Asynchronously switched control of switched linear systems with average dwell time [J].Automatica, vol. 46, pp. 953-958, 2010.
[7] Z. Ke, J. Bin. Analysis and design of adaptive fault estimation for timevarying delay systems [J]. pp. 38-42.2008.

[8] D. Dongsheng. Fault diagnosis for switched systems with time delay [J]., pp. 2162-2165.2010. 